\title{
The Satellite's On-Orbit Attitude System Error Compensation Technique Based on Stereo Models
}

\author{
Yu Zhou 1,2,3, Xin Hu ${ }^{1,2,3}$, Peng Du ${ }^{2,3}$, Yi Wang4 \\ ${ }^{1}$ Information Engineering University, Zhengzhou, China \\ ${ }^{2}$ State Key Laboratory of Geo-Information Engineering, Xi'an, China \\ ${ }^{3}$ Xi'an Research Institute of Surveying and Mapping, Xi'an, China \\ ${ }^{4}$ ZhongkeXingtu Co., Ltd., Xi'an, China \\ Email: hb48_zy@163.com
}

How to cite this paper: Zhou, Y., Hu, X., Du, P. and Wang, Y. (2020) The Satellite's On-Orbit Attitude System Error Compensation Technique Based on Stereo Models. Journal of Computer and Communications, 8, 42-53.

https://doi.org/10.4236/jcc.2020.86005

Received: June 5, 2020

Accepted: June 27, 2020

Published: June 30, 2020

\begin{abstract}
According to the problem that the low measurement accuracy of TH-1 satellite star sensor, the low frequency and "slow drift" error which cannot be ignored in the attitude determination system, resulting in obvious random error in the horizontal position and elevation direction, and the change of the error with time and latitude, cannot be calibrated by the ground field of the real problem. In this paper, a low frequency detection model is established by using the principle of relative orientation, and the low frequency error is obtained by parallax elimination. Finally, the satellite attitude is compensated and the more accurate exterior orientation elements are obtained, thus improving the positioning accuracy and stability. The experimental results show that: the proposed methods are feasible, and by using the model to dynamically calibrate the exterior orientation angle elements on orbit, the plane and elevation errors of the ground points can be basically eliminated. The global uncontrollable positioning accuracy and stability of the photogrammetry satellite are improved.
\end{abstract}

\section{Keywords}

Attitude Measuring System, Low Frequency Error, Dynamic Calibration, Relative Orientation

\section{Introduction}

It is an important problem that high-precision positioning accuracy without GCPs, which is plaguing the acquisition of high-precision geographic information 
products around the world [1] [2] [3]. Research status and engineering practices show that the high positioning accuracy of satellite imagery has little to do with image resolution [1]. The key is the acquisition of high-precision attitude data. If the external orientation elements of the satellite on-orbit detection are highly accurate and reliable, then it is simple to realize the global photogrammetry problem without GCPs [4] [5]. The geometric calibration technique is used to calibrate the error of the external orientation elements in the calibration field [6] [7]. In this way, the requirements for positioning without GCPs can be achieved. However, due to the low accuracy of satellite star sensors such as TH-1 and ZY-3, the low frequency errors and "slow drift" errors are not negligible, which will result in significant random errors in the horizontal and altitude directions [8] [9]. Different from the system error, the error of the attitude measurement system changes with time and latitude, resulting in its short-period systematization in the horizontal position direction [10]. The static calibration in orbit cannot give a stable solution. It causes great difficulties in positioning without ground control points (GCPs) [11].

The TH-1 satellite adopts the unique EFP (Equivalent Frame Photo) bundle adjustment, and the low-frequency error compensation technology is proposed on this basis, which realizes the global uncontrolled high-precision positioning, achieving the positioning accuracy of $3.7 \mathrm{~m}$ on plane and $2.4 \mathrm{~m}$ on elevation which meets the mapping accuracy requirement of 1:25000 geographic information product [12]. EFP bundle adjustment is based on the special three-line-array LMCCD aerospace surveying camera system; the low-frequency error compensation with this method is not reported in other satellite projects. In addition, in the domestic study on the positioning accuracy of ZY-3, a few GCPs are needed to fit the system error and improve the positioning accuracy [13].

In this paper, our work is spirited by above method, we use the vertical parallax between the stereo image pair to eliminate the system error of image's pitch angle and yaw angle, and then the relative orientation of two images is restored. The positioning accuracy of satellite images is improved by compensating low frequency error of the pitch angle and yaw angle. The proposed model is simple to be constructed and its transplantation to the general aerospace photogrammetry satellite positioning method is convenient.

The low-frequency error compensation model and compensation process are also given in this paper. The proposed method is tested by the actual data of TH-1 satellite, which proves its correctness and feasibility [14].

\section{Low-Frequency Error Compensation Model for Exterior Orientation Angle Elements}

\subsection{Fundamental Theory}

By using the principle of relative orientation, the low frequency detection model is established, and the low frequency error of attitude is obtained by eliminating parallax. The satellite attitude is compensated to obtain more accurate foreign position elements and improve the accuracy of satellite positioning. 
As shown in Figure 1, $a 1$ and $a 2$ are a pair of homonymy light, homonymy light pair is located in the same epipolar plane as the photographic baseline $B$, that is, $a 1, a 2$ and $B$ three lines are coplanar. From the knowledge of spatial analytic geometry, if three lines are coplanar, the mixed product of their corresponding vectors is zero, shown as (1) [15].

$$
B \cdot(a 1 \times a 2)=0
$$

\subsection{The Basic Calculation Process}

The coordinates of the three vectors in the image space assisted coordinate system are respectively $\left(B_{x}, B_{y}, B_{z}\right),\left(X_{1}, Y_{1}, Z_{1}\right)$ and $\left(X_{2}, Y_{2}, Z_{2}\right)$, The coplanar condition equation can be represented by coordinates as (2).

$$
F=\left|\begin{array}{lll}
B_{x} & B_{y} & B_{z} \\
X_{1} & Y_{1} & Z_{1} \\
X_{2} & Y_{2} & Z_{2}
\end{array}\right|=0
$$

Whether the coplanar condition equation is established is the standard to complete the relative orientation. The analytic relative orientation is to solve elements of relative orientation according to the coplanar condition equation. When relative directional parsing is calculated, the photographic baseline $B$ is usually rewritten to $b$, which is called projection baseline. Here,

$$
B=m \cdot b
$$

where $m$ is the photographic scale denominator; $\left(b_{x}, b_{y}, b_{z}\right)$ are the components of the projection baseline. From the geometric relationship in Figure 1, we can get (4)

$$
\left\{\begin{array}{c}
b_{y}=b_{x} \cdot \tan \mu \approx b_{x} \cdot \mu \\
b_{z}=\frac{b_{x}}{\cos \mu} \cdot \tan v \approx b_{x} \cdot v
\end{array}\right.
$$

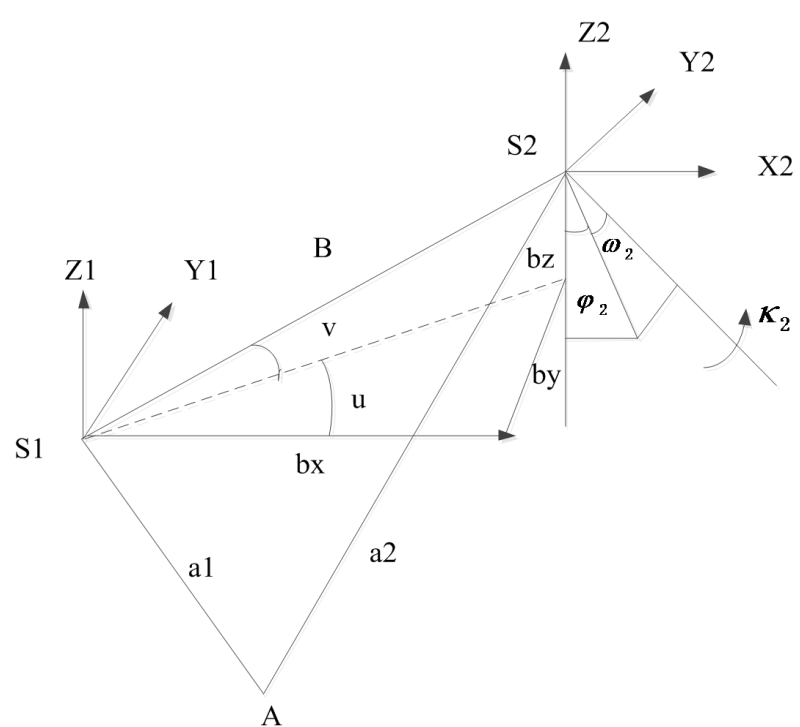

Figure 1. Principle of relative orientation. 
in (4), $\mu$ is the deflection angles of the baseline (that is, the yaw corner of low frequency error) and $v$ is the inclination (that is, the pitch angle of low frequency error). Substituting the above formula into the coplanar condition equation.

$$
F=\left|\begin{array}{ccc}
b_{x} & b_{x} \mu & b_{x} v \\
X_{1} & Y_{1} & Z_{1} \\
X_{2} & Y_{2} & Z_{2}
\end{array}\right|=b_{x}\left|\begin{array}{ccc}
1 & \mu & v \\
X_{1} & Y_{1} & Z_{1} \\
X_{2} & Y_{2} & Z_{2}
\end{array}\right|=0
$$

$F$ will be expanded by the Taylor series, holding the first order terms, and the linear formula of the coplanar equation is

$$
F=F_{0}+\frac{\partial F}{\partial \mu} d \mu+\frac{\partial F}{\partial v} d v+\frac{\partial F}{\partial \phi} d \phi+\frac{\partial F}{\partial \omega} d \omega+\frac{\partial F}{\partial \kappa} d \kappa=0
$$

the derivation is finally available:

$$
Q=b_{x} d \mu-\frac{Y_{2}}{Z_{2}} b_{x} d v-\frac{X_{2} Y_{2}}{Z_{2}} N_{2} d \phi-\left(Z_{2}+\frac{Y_{2}^{2}}{Z_{2}}\right) N_{2} d \omega+X_{2} N_{2} d \kappa
$$

the upper formula is converted into an error equation:

$$
V_{Q}=b_{x} d \mu-\frac{Y_{2}}{Z_{2}} b_{x} d v-\frac{X_{2} Y_{2}}{Z_{2}} N_{2} d \varphi-\left(Z_{2}+\frac{Y_{2}^{2}}{Z_{2}}\right) N_{2} d \omega+X_{2} N_{2} d \kappa-Q
$$

where $X_{2}, Y_{2}, Z_{2}$ is the coordinate of homonymy points in right image in the photogrammetry coordinate system, $b_{x}$ represents the component of baseline $b$ in the Axis $X, N_{2}$ represents the right image projection coefficient, $Q$ is the vertical parallax. $\mu, v$ represent the low-frequency detection error values. The correction amount of attitude in pitch and yaw direction is calculated by global matching connection point, and the error compensation is carried out.

\section{Provisions for Changes in the Inner Orientation Elements of Camera}

Taking the TH-1 satellite as an example, the three-line-array CCD space photogrammetry camera adopts the form of three-lens three-line array, and the three-lens three-line array constitutes an image plane equivalent to the same frame, with three CCD linear array spaces parallel and horizontally placed. That is, the on-orbit calibration based on the change values of the inner orientation elements is under the assumption that the image plane is horizontal.

However, in the long flight, the vibration and change of temperature of satellite platform will affect the three-line-array CCD camera's change, it causes the virtual image plane is not horizontal, showing the change that the tilt up and down.

As shown in Figure 2, the tilt error $\alpha_{v}$ of the three-line-array image plane:

It can be seen from Figure 2 that, the tilt error of the three-line-array image plane, the pitch angle error of low-frequency measurement of star sensor and the pitch angle error of camera in camera body coordinate system (the system error on pitch angle direction of the angle between star sensor and earth observation 


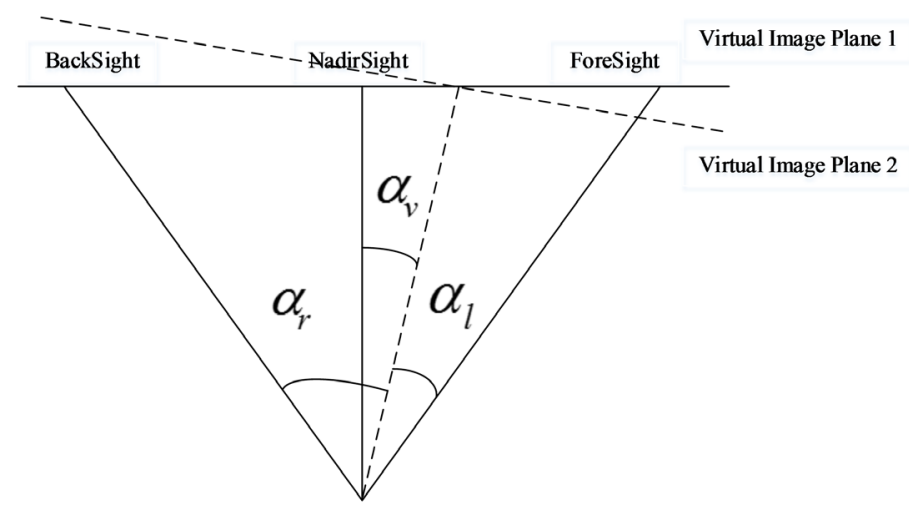

Figure 2. Tilt up and down of the virtual image plane's diagrammatic drawing.

camera, referred to as the pitch angle error) is all in the same expression form. Therefore, when the angle between star sensor and earth observation cameras geometrically corrected, the tilt error of the three-line-array image plane and low-frequency measurement error will be confused with pitch angle error. When the low-frequency error of the exterior orientation angle elements is compensated, the tilt error of the three-line image plane will be confused to low-frequency measurement error.

In fact, the tilt error of the three-line-array image plane, the pitch angle error and the low-frequency measurement error are different in mechanism, and the error properties are different.

Repeated detection or leak detection without reasonable discrimination will result in lower ground positioning accuracy. Research shows that the tilt error of the three-line image plane is up to tens of seconds, and its influence on the positioning accuracy cannot be ignored.

Therefore, when there is the tilt error of three-line-array image plane, in order to avoid repeated detection or leak detection, it is necessary to re-specify the inner orientation elements in the form of

$$
\left\{\begin{array}{c}
f_{l}=F_{l} \cdot \cos \left(\alpha_{l}+\alpha_{v}\right) \\
x_{l}=f_{l} \cdot \tan \left(\alpha_{l}+\alpha_{v}\right) \\
f_{v}=F_{v} \\
x_{v}=0 \\
f_{r}=F_{r} \cdot \cos \left(\alpha_{r}-\alpha_{v}\right) \\
x_{r}=-f_{l} \cdot \tan \left(\alpha_{l}-\alpha_{v}\right)
\end{array}\right.
$$

\section{Low Frequency Error Correction Solution}

\subsection{The Correct Model of $d \mu, d v$}

In the error Equation (8), the vertical parallax of homonymy points originates not only from the system value produced by the low frequency error of the attitude measurement system, but also from the accidental value generated by the random error of the attitude measurement system. 
In the actual low frequency error compensation, using vertical parallax of all points participating in the adjustment as the basis, the random error can be weakened by the adjustment method (about 0.6 factors) and its size can be ignored, that is, $d \varphi, \mathrm{d} \omega$ and $\mathrm{d} \kappa$ are about zero.

At the same time, centering on the central axis of the flight direction of strip image, match two rows of homonymy points on the upper and lower sides of the same strip image for constraining. Taking one pair of homonymy points in the upper and lower rows as an example, the vertical parallax can be converted to

$$
\left\{\begin{array}{l}
Q_{U}=b_{x} d \mu_{U}-\frac{Y_{2 U}}{Z_{2 U}} b_{x} d v_{U} \\
Q_{D}=b_{x} d \mu_{D}-\frac{Y_{2 D}}{Z_{2 D}} b_{x} d v_{U}
\end{array}\right.
$$

In the equation the symbol $U$ and $D$ respectively represents the upper and lower sides of the same strip image for constraining. Because of the upper and lower symmetry of the homonymy points, there is

$$
\left\{\begin{array}{c}
\mathrm{x}_{U}=x_{D}, \quad \mathrm{y}_{U}=-y_{D} \\
d \mu_{U}=d \mu_{D}, \quad d v_{U}=d v_{D}
\end{array}\right.
$$

bring Equation(11) into the Equation (12)

$$
\left[\begin{array}{c}
\mathrm{X}_{2} \\
\mathrm{Y}_{2} \\
\mathrm{Z}_{2}
\end{array}\right]=\mathrm{m} R\left[\begin{array}{c}
x \\
y \\
-f
\end{array}\right]
$$

we can get $X_{2 U}=X_{2 D}, Y_{2 U}=-Y_{2 D}, Z_{2 U}=Z_{2 D}$.

The vertical parallax of the homonymy points is further transformed into

$$
\left\{\begin{array}{l}
Q_{U}=b_{x} d \mu_{U}-\frac{Y_{2 U}}{Z_{2 U}} b_{x} d v_{U}=b_{x} d \mu+\frac{Y_{2}}{Z_{2}} b_{x} d v \\
Q_{D}=b_{x} d \mu_{D}-\frac{Y_{2 D}}{Z_{2 D}} b_{x} d v_{D}=b_{x} d \mu-\frac{Y_{2}}{Z_{2}} b_{x} d v
\end{array}\right.
$$

1) The special correction of $d \mu$ available by Formula (14) (Figure 3)

$$
\mathrm{Q}_{U}+\mathrm{Q}_{D}=2 b_{x} d \mu
$$

the correction value of the obtained $d \mu$ is

$$
d \mu=\frac{\mathrm{Q}_{U}+\mathrm{Q}_{D}}{2 b_{x}}
$$

2) The special correction of $d v$ available by Formula (16) (Figure 4):

$$
\mathrm{Q}_{U}-\mathrm{Q}_{D}=2 \frac{Y_{2}}{Z_{2}} b d v
$$

the correction value of the obtained $d v$ is:

$$
d v=\frac{\mathrm{Q}_{U}-\mathrm{Q}_{D}}{2 \mathrm{Y}_{2}} \mathrm{Z}_{2}
$$




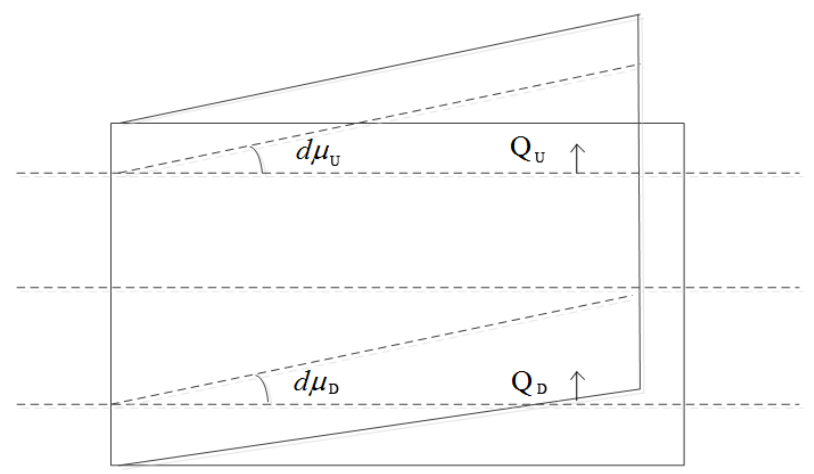

Figure 3. The special correction of $d \mu$.

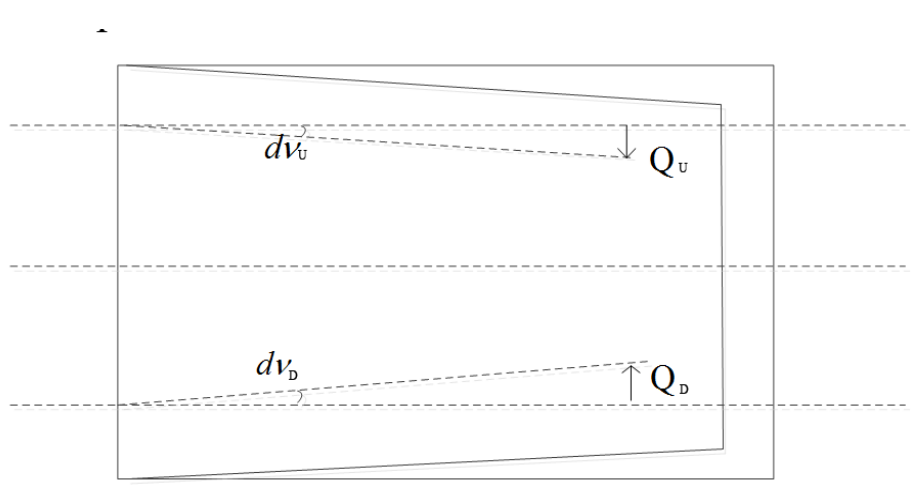

Figure 4. The special correction of $d v$.

\subsection{Low Frequency Error Compensation Process}

The process of low frequency error compensation is as follows and the for details can be seen in Figure 5.

Step 1: Fully automatic match the three-line-array image, and obtain the image points coordinates of the upper and lower rows on the forward-looking, nadir-looking and backward-looking images.

Step 2: Coordinate transformation of the original pose data, and convert attitude data between CGCS2000 and earth observation camera to the local and camera coordinate system for the vertical parallax calculation.

Step 3: According to the relative orientation principle, calculate the parallax of the upper and lower rows points, and the correction amount corresponding to $d \mu$ of each row, and use the sieve strategy to eliminate the outlier to ensure the uniformity of the upper and lower parallax distribution of the upper and lower rows points. Thus, the average values of $d \mu$ and $d v$ are obtained.

Step 4: Correct $d \mu$ and $d v$, and calculate the parallax again, check whether it meets the threshold.

Step 5: After the correction is completed, the pose data is converted to the data between CGCS2000 and the camera.

Step 6: Use the corrected attitude data for bundle adjustment and assess the positioning accuracy. 


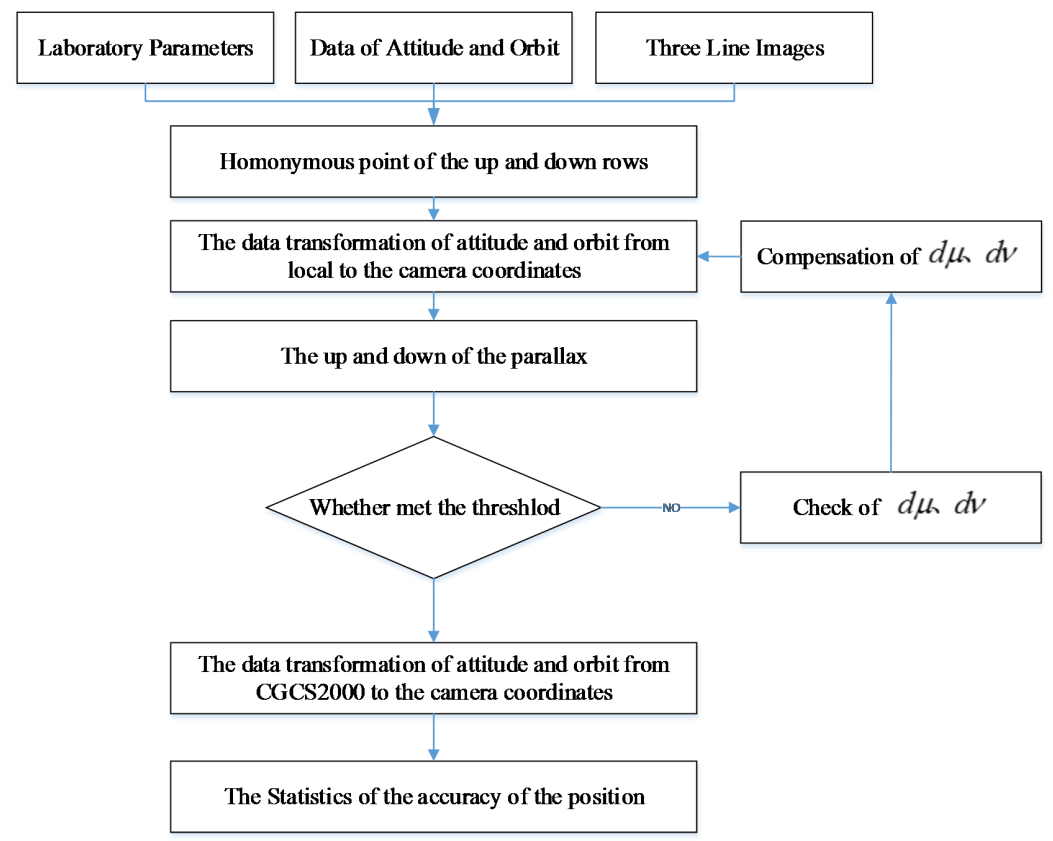

Figure 5. The flow diagram of low frequency compensation.

\section{Experiments}

\subsection{Sensitivity Analysis of Low Frequency Error Compensation}

In this paper, we used five test sites of TH01-01 and TH01-02's image to validate our methods. After static on-orbit geometry inspection of five test sites of TH01-01 star and TH01-02 star, the system error is basically eliminated, that is, when each test site star sensor data is corrected by its own calibration parameters, the low frequency measurement error should be eliminated. Under the condition of tilt error compensation of three-line-array image plane, the low frequency error of five test sites should theoretically be 0 .

The low frequency error detection of TH01-01 star and TH01-02 star is obtained by means of dynamic calibration technique of exterior orientation angle elements, and the low-frequency error correction value is given as shown in Table 1.

1) The pitch angle and yaw angle error of each test site fluctuates around 0 , showing randomness, through the error calculation, the sensitivity of low-frequency error compensation is nearly $1 "$, which is a high value.

2) The sensitivity of yaw angle is higher than the pitch angle, and its value is basically zero. Through the position method of the satellite images, the system error of the yaw angle will cause elevation accuracy error [14]. Therefore, the reduction of elevation accuracy caused by low-frequency measurement error can be basically eliminated by dynamic calibration.

\subsection{Correction of Tilt Error of Third Line Array Image Plane}

After static on-orbit geometry calibration, the real pitch angle and yaw angle correction values of the same test sites are obtained by the dynamic calibration technique of exterior orientation angle elements, which is shown in Table 2. 
Table 1. The result of the TH-01 and TH-02 satellites' dynamic calibration. ( $d v$ : dynamiccalibration value of pitch angle, $d \mu$ : dynamic calibration value of yaw angle, unit: seconds).

\begin{tabular}{ccccc}
\hline \multirow{2}{*}{ Test sites } & \multicolumn{2}{c}{$\begin{array}{c}\text { Dynamic calibration } \\
\text { value of TH01 }\end{array}$} & \multicolumn{2}{c}{$\begin{array}{c}\text { Dynamic calibration } \\
\text { value of TH-02 }\end{array}$} \\
\cline { 2 - 5 } & $d V$ & $d \mu$ & $d V$ & $d \mu$ \\
\hline Xinjiang & 0.45 & -0.59 & -0.69 & 0.12 \\
Beijing & -0.77 & 0.13 & 1.4 & -0.05 \\
Heilongjiang & 0.91 & -0.09 & -0.12 & 0.38 \\
Anhui & 0.15 & 0.54 & 1.13 & 0.27 \\
Heilongjiang and Jilin & 1.42 & -0.11 & -1.98 & -0.42 \\
RMS & 0.86 & 0.37 & $\mathbf{1 . 2 4}$ & 0.29 \\
\hline
\end{tabular}

Table 2. The result of the TH-01 and TH-02 satellites' dynamic calibration. ( $d v, d \mu$ : dynamic calibration value of pitch and yaw angle, unit: seconds).

\begin{tabular}{ccccc}
\hline \multirow{2}{*}{ Test sites } & \multicolumn{2}{c}{$\begin{array}{c}\text { Dynamic calibration } \\
\text { value of TH01 }\end{array}$} & \multicolumn{2}{c}{$\begin{array}{c}\text { Dynamic calibration } \\
\text { value of TH-02 }\end{array}$} \\
\cline { 2 - 5 } & $d V$ & $d \mu$ & $d V$ & $d \mu$ \\
\hline Xinjiang & 2.36 & -0.58 & 9.77 & 0.02 \\
Beijing & 1.22 & 0.12 & 12.21 & -0.05 \\
Heilongjiang & 2.92 & -0.13 & 10.42 & 0.38 \\
Anhui & 2.18 & 0.48 & 11.65 & 0.19 \\
Heilongjiang and Jilin & 3.34 & -0.21 & 8.54 & -0.38 \\
RMS & 2.51 & $\mathbf{0 . 3 6}$ & $\mathbf{1 0 . 6 0}$ & $\mathbf{0 . 2 6}$ \\
\hline
\end{tabular}

1) The pitch angle error of TH01-01 star and TH01-02 star is systematic, the error of TH01-01 star's test sites fluctuates around the mean value 2.51", and the error of TH01-01 star's test sites revolves around the mean value 10.60".

2) The partial system error of the pitch angle of TH01-01 star and TH01-02 star is the tilt error of the three-line-array image plane.

3) After correcting the tilt error of the three-line-array image plane of TH01-01 star and TH01-02 star, the real low-frequency measurement error can be obtained by dynamic calibration, as shown in Table 2 .

\subsection{Low Frequency Compensation Performance Verification}

After dynamic calibration of the test site data, the measurement error of the star sensor is corrected, and the positioning without GCPs is carried out. The positioning accuracy results of TH01-01 star and TH01-02 star are shown as Tables 3-5.

Comparing the experimental results above, we can summarize that:

1) Because of the existence of low-frequency measurement error, the positioning accuracy without GCPs of each test site corrected by the test parameters of Beijing calibration field is lower than the accuracy of each test site corrected by the calibration parameters of each test site. 
Table 3. The positioning accuracy without GCPs in each site verified by Beijing calibration field parameters ( $X, Y$ : Gaussian projection, $H$ : elevation, unit: meters).

\begin{tabular}{ccccccccc}
\hline \multirow{2}{*}{ Test sites } & \multicolumn{4}{c}{ TH01-01 star } & \multicolumn{5}{c}{ TH01-02 star } \\
\cline { 2 - 8 } & $\mathrm{X}$ & $\mathrm{Y}$ & $\mathrm{XY}$ & $\mathrm{H}$ & $\mathrm{X}$ & $\mathrm{Y}$ & $\mathrm{XY}$ & $\mathrm{H}$ \\
\hline Xinjiang & 14.06 & 11.31 & 18.04 & 6.85 & 8.35 & 10.03 & 13.05 & 10.28 \\
Beijing & 6.58 & 5.28 & 8.44 & 5.88 & 6.42 & 8.12 & 10.35 & 10.56 \\
Heilongjiang & 11.83 & 11.24 & 16.32 & 7.48 & 6.77 & 9.22 & 11.44 & 8.75 \\
Anhui & 7.42 & 6.28 & 9.72 & 8.45 & 9.12 & 9.98 & 13.52 & 11.12 \\
Heilongjiang and & 13.33 & 12.14 & 18.10 & 8.34 & 4.23 & 9.78 & 10.66 & 10.35 \\
Jilin & & & & & & & & \\
RMS & $\mathbf{1 1 . 0 8}$ & $\mathbf{9 . 6 8}$ & $\mathbf{1 4 . 7 1}$ & $\mathbf{7 . 4 6}$ & $\mathbf{7 . 1 8}$ & $\mathbf{9 . 4 5}$ & $\mathbf{1 1 . 8 7}$ & $\mathbf{1 0 . 2 4}$ \\
\hline
\end{tabular}

Table 4. The positioning accuracy without GCPs in each site verified by each test site parameters ( $X, Y$ : Gaussian projection, $H$ : elevation, unit: meters).

\begin{tabular}{ccccccccc}
\hline \multirow{2}{*}{ Test sites } & \multicolumn{4}{c}{ TH01-01 star } & \multicolumn{5}{c}{ TH01-02 star } \\
\cline { 2 - 9 } & $\mathrm{X}$ & $\mathrm{Y}$ & $\mathrm{XY}$ & $\mathrm{H}$ & $\mathrm{X}$ & $\mathrm{Y}$ & $\mathrm{XY}$ & $\mathrm{H}$ \\
\hline Xinjiang & 4.28 & 5.79 & 7.20 & 6.89 & 6.96 & 5.89 & 9.12 & 5.12 \\
Beijing & 3.12 & 5.38 & 6.22 & 5.63 & 7.28 & 6.12 & 9.51 & 10.87 \\
Heilongjiang & 3.45 & 4.12 & 5.37 & 5.12 & 3.55 & 3.39 & 4.91 & 3.87 \\
Anhui & 2.98 & 3.09 & 4.29 & 7.41 & 3.38 & 5.12 & 6.14 & 4.28 \\
Heilongjiang and & 5.97 & 4.88 & 7.71 & 6.12 & 4.50 & 4.67 & 6.49 & 2.89 \\
Jilin & & & & & & & & \\
RMS & $\mathbf{4 . 1 1}$ & $\mathbf{4 . 7 5}$ & $\mathbf{6 . 2 8}$ & $\mathbf{6 . 2 9}$ & $\mathbf{5 . 4 0}$ & $\mathbf{5 . 1 3}$ & $\mathbf{7 . 4 5}$ & $\mathbf{6 . 1 0}$ \\
\hline
\end{tabular}

Table 5. The positioning accuracy without GCPs after low-frequency error compensation ( $X, Y$ : Gaussian projection, $H$ : elevation, unit: meters).

\begin{tabular}{ccccccccc}
\hline \multirow{2}{*}{ Test sites } & \multicolumn{3}{c}{ TH01-01 star } & \multicolumn{5}{c}{ TH01-02 star } \\
\cline { 2 - 9 } & $\mathrm{X}$ & $\mathrm{Y}$ & $\mathrm{XY}$ & $\mathrm{H}$ & $\mathrm{X}$ & $\mathrm{Y}$ & $\mathrm{XY}$ & $\mathrm{H}$ \\
\hline Xinjiang & 6.12 & 6.09 & 8.63 & 5.12 & 7.12 & 6.12 & 9.39 & 5.12 \\
Beijing & 3.22 & 5.80 & 6.63 & 6.02 & 10.02 & 5.48 & 11.42 & 10.33 \\
Heilongjiang & 4.89 & 7.31 & 8.79 & 4.83 & 4.12 & 6.23 & 7.47 & 4.08 \\
Anhui & 3.12 & 6.97 & 7.64 & 5.21 & 3.09 & 6.04 & 6.78 & 3.99 \\
Heilongjiang and & 5.89 & 4.30 & 7.29 & 6.89 & 6.89 & 5.87 & 9.05 & 2.78 \\
Jilin & & & & & & & & \\
RMS & $\mathbf{4 . 8 2}$ & $\mathbf{6 . 1 8}$ & $\mathbf{7 . 8 4}$ & $\mathbf{5 . 6 6}$ & $\mathbf{6 . 7 1}$ & $\mathbf{5 . 9 5}$ & $\mathbf{8 . 9 7}$ & $\mathbf{5 . 8 9}$ \\
\hline
\end{tabular}

2) Correct the low-frequency measurement error by dynamically calibration, the positioning accuracy of plane and elevation is improved, especially the elevation accuracy can be improved to the accuracy of each test site corrected by the calibration parameters of each test site.

3) Correct low-frequency measurement error by dynamic calibration, and the plane positioning accuracy is still lower than the accuracy of each test sites corrected by the calibration parameters of each test site. 


\section{Conclusion}

Through the technique of exterior orientation angle elements dynamic calibration, the low-frequency error detection of TH01-01 star and TH01-02 star can basically eliminate the plane and elevation error of the ground points, and the sensitivity of the low-frequency error compensation is about 1", which is a high sensitivity. Under the condition of low precision of domestic satellite attitude measurement system, it is of great significance to the improvement of global positioning accuracy without GCPs.

\section{Conflicts of Interest}

The authors declare no conflicts of interest regarding the publication of this paper.

\section{References}

[1] Wang, R.X., Wang, J.X. and Hu, X. (2016) Preliminary Location Accuracy Assessments of 3rd Satellite of TH-1. Acta Geodaeticaet Cartographica Sinica, 45, 1135-1139.

[2] Zhang, Y., Zheng, M., Xiong, X., et al. (2014) Multistrip Bundle Block Adjustment of ZY-3 Satellite Imagery by Rigorous Sensor Model without Ground Control Point. IEEE Geoscience and Remote Sensing Letters, 12, 865-869. https://doi.org/10.1109/LGRS.2014.2365210

[3] Zhang, G., Jiang, Y.H., Li, D.R., Huang, W.C. and Pan, H.B. (2014) In-Orbit Geometric Calibration and Validation of ZY-3 Linear Array Sensors. The Photogrammetric Record, 29, 68-88. https://doi.org/10.1111/phor.12052

[4] Jeong, J. and Kim, T. (2015) Comparison of Positioning Accuracy of a Rigorous Sensor Model and Two Rational Function Models for Weak Stereo Geometry. ISPRS Journal of Photogrammetry and Remote Sensing, 108, 172-182. https://doi.org/10.1016/j.isprsjprs.2015.07.006

[5] Aguilar, M.A., Aguilar, F.J., Saldañ, M., et al. (2012) Geopositioning Accuracy Assessment of GeoEye-1 Panchromatic and Multispectral Imagery. Photogrammetric Engineering \& Remote Sensing, 78, 247-257. https://doi.org/10.14358/PERS.78.3.247

[6] Jiang, Y.H., Zhang, G., Tang, X.M., Li, D.R., Huang, W.C. and Pan, H.B. (2014) Geometric Calibration and Accuracy Assessment of ZiYuan-3 Multispectral Images. IEEE Transactions on Geoscience and Remote Sensing, 52, 4161-4172. https://doi.org/10.1109/TGRS.2013.2280134

[7] Wang, J.R., Wang, R.X., Hu, X. and Su, Z.B. (2017) The On-Orbit Calibration of Geometric Parameters of the Tian-Hui 1 (TH-1) Satellite. ISPRS Journal of Photogrammetry and Remote Sensing, 124, 144-151. https://doi.org/10.1016/j.isprsjprs.2017.01.003

[8] Wang, R.X., Wang, J.R. and Hu, X. (2016) Low-Frequency Errors Compensation of Attitude Determination System in Satellite Photogrammetry. Acta Geodaeticaet Cartographica Sinica, 45, 127-130.

[9] Guan, Z.C., Jiang, Y.H., Wang, J.Y. and Zhang, G. (2019) Star-Based Calibration of the Installation Between the Camera and Star Sensor of the Luojia 1-01 Satellite. Remote Sensing, 11, 2081. https://doi.org/10.3390/rs11182081

[10] Jiang, Y.H., Zhang, G., Li, D.R., Tang, X.M., Huang, W.C. and Li, L.T. (2015) Correction of Distortions in YG-12 High-Resolution Panchromatic Images. Photogram- 
metric Engineering \& Remote Sensing, 81, 25-36.

https://doi.org/10.14358/PERS.81.1.25

[11] Wang, J.R., Wang, R.X. and Hu, X. (2018) Self-Adaption Bundle Adjustment of Three-Line Array Image with Smoothing Equation of Exterior Orientation Elements. Acta Geodaeticaet Cartographica Sinica, 47, 968-972.

[12] Wang, R.X., Wang, J.R., Li, J., Zhu L.M., Li, W. and Yang, J.F. (2019) Improvement Strategy for Location Accuracy without Ground Control Points of 3rd Satellite of TH-1. Acta Geodaeticaet Cartographica Sinica, 48, 671-675.

[13] Jiang, Y.H., Zhang, G., Li, D.R., Huang, W.C. andLi, L.T. (2015) Improvement and Assessment of the Geometric Accuracy of Chinese High-Resolution Optical Satellites. IEEE Journal of Selected Topics in Applied Earth Observations and Remote Sensing, 8, 4841-4852. https://doi.org/10.1109/JSTARS.2015.2429151

[14] Wang, R.X. (2016) Satellite Photogrammetric Principle for Three-Line-Array CCD Imagery. Surveying and Mapping Press, Beijing.

[15] Wang, Z.Z. (1990) Principle of Photogrammetry. Publishing House of Surveying and Mapping, Beijing. 